\title{
PROPOSED ADDITION TO THE "OFFICIAL LIST OF GENERIC NAMES IN ZOOLOGY" OF THE NAMES OF ONE HUNDRED AND TWO GENERA OF CARIDEA (CLASS CRUSTACEA, ORDER DECAPODA), INCLUDING PROPOSALS FOR THE USE OF THE PLENARY POWERS (a) TO VALIDATE THE EMENDATION TO "GNATHOPHYLLUM" OF THE GENERIC NAME "GNATO- PHYLLUM" LATREILLE, 1814, AND (b) TO VALIDATE THE FAMILY-GROUP NAMES “ HIPPOLYTIDAE” BATE, 1888 AND " EUGONATONOTIDAE" CHACE, 1937
}

\author{
By L. B. HOLTHUIS \\ (Rijksmuseum van Natuurlijke Historie, Leiden, The Netherlands)
}

(Commission's Reference : Z.N.(S.) 622)

I submit herewith to the International Commission on Zoological Nomenclature a list of the names of one hundred and two genera of Caridean Crustacea for addition to the Official List of Generic Names in Zoology. Each of these names is an available name in the sense that it is not a homonym of any generic name previously published for a genus in the Animal Kingdom. All of these names are currently used in carcinological literature. The validity of only one among them has been questioned. This one case is dealt with in paragraph 2(1) below. The species indicated here as type species of the genera enumerated in the list have been duly determined as such under the Règles Internationales de la Nomenclature Zoologique. The gender of each generic name is indicated in brackets immediately after the name concerned:

2. A few cases call for some special comments, which are given here in a separate paragraph before the actual list. These cases are set out below :-

(1) Argis Kröyer, 1842: The name Argis Kröyer, 1842, is rejected by some authors on account of Arges de Haan, 1833. According to the Règles the difference between the two names is sufficient to let Argis Kröyer stand as a valid name (1953, Copenhagen Decisions Zool. Nomencl. : 78, Decision 152). The authors who reject Argis Kröyer, use in its place the generic name Nectocrangon Brandt, 1851 (Middendorff's Reise Sibir. 2(1) : 114) (type species, by monotypy: Crangon lar Owen, 1839, Zool. Beechey's Voy. Blossom: 88). Argis Kröyer and Nectocrangon Brandt are objective synonyms since they have the same type species. The name Nectocrangon Brandt, 1851, being an 
invalid junior objective synonym of Argis Kröyer, 1842, should be placed on the Official Index of Rejected and Invalid Generic Names in Zoology at the same time that Argis Kröyer, 1842, is placed on the Official List of Generic Names in Zoology.

(2) Caridion Goës, 1863: In the first description of the type species of this genus, Bate (1858, Nat. Hist. Rev. Proc. Soc. Dublin $5: 51,52)$ did not give the species a scientific name, though he stated that he would name it for a Mr. Gordon. The name Hippolyte gordoni is only mentioned on p. iv. of the index of the fifth volume of Nat. Hist. Rev. Proc. Soc. Dublin, which contains Bate's paper. That Bate is not the author of this name Hippolyte gordoni is the more probable since in the following year he published a description of the same species in Proc. Dublin Univ. zool. bot. Ass. $(1: 48,49)$ under the name Hippolyte gordoniana. However, there can be no certainty on this point. As the specific name gordoni, which at present is universally used for the species, has always been attributed to Bate, it seems best to continue this practice and to consider Hippolyte gordoni Bate, 1858, the oldest available name for the species discussed here.

(3) Chorismus Bate, 1888: In the original description of Chorismus, Bate dealt only with $C$. tuberculatus, but he stated at the end of the text concerning the genus: "Tozeuma serratum, A. Milne Edwards, ... belongs, I think, to this genus ". The qualified expression so used by Bate appears to me to imply that he only doubtfully included this species in his genus. On this interpretation Tozeuma serratum is excluded by Rule (e) in Article 30 from consideration as a possible type species for the genus Chorismus. I therefore consider that C. tuberculatus should be regarded as being the type species by monotypy. If it were to be held that by the remark quoted above Bate included T. serratum in the genus Chorismus, that genus would not be monotypical. In that case, Chorismus tuberculatus would still, however, be the type species of this genus, it having been so selected by Calman in 1907 (Nat. Antarct. Exped., Nat. Hist. 2 (Crust.)(1) : 3).

(4) Dorodotes Bate, 1888: In the original description of the genus in 1888 Bate stated on p. 677: "I only know of one species of this genus and this has been taken twice, in the Sea of Banda and near Manila". However, he actually described two species in the genus : D. reflexus from the Banda Sea and Manila, and D. levicarina from the Arafura Sea. It seems probable that, when Bate first drew up the description of this genus, he only referred $D$. reflexus to it and that it was only at some later stage he inserted $D$. levicarina also. I do not think that 
this evidence is sufficient to indicate $D$. reflexus as the type species of the genus by original designation. De Man (1920, Siboga Exped. $39(\mathrm{a} 3)$ : 151), after having placed Dorodotes levicarina Bate in a separate genus, remarked: "The genus Dorodotes Bate is still only represented by one species ", but this also cannot be regarded as an unequivocal type selection.

(5) Gelastocaris Kemp, 1914: The specific name of the nominal species Latreutes paronai Nobili, the type species of the genus Gelastocaris, was originally written by Nobili as paronae. Since the species was named after Professor C. Parona, who is a man and not a woman, the original orthography paronae is incorrect and is subject to automatic correction to paronai.

(6) Gnathophyllum Latreille, 1819: Latreille's original spelling of this name was Gnatophyllum. The name was emended by Desmarest, 1823 (Dict. Sci. nat. $28: 322$ ) to Gnathophyllum. The emended name has been accepted by practically all subsequent authors, among them, Latreille himself, and at present it is in general use. The Commission therefore is asked to use its Plenary Powers to validate the emended spelling Gnathophyllum and, having done so, to place this name so emended on the Official List and the original spelling Gnatophyllum on the Official Index of Rejected and Invalid Generic Names in Zoology.

(7) Hippolyte Leach, 1814: In the original description of this genus Leach remarked: "To this genus Cancer astacus gibbosus of Montagu, already referred to in the note after Penaeus, page 401, belongs". This statement is quite unequivocal and should therefore be interpreted as meaning that he placed two nominal species in this genus, namely C. gibbosus Montagu as well as Hippolyte varians. On this view this genus is not monotypical. The second of these species was, however, selected as type species by Leach himself in 1817 (Malac. podophth. Brit. (16) : first page of explanation for pl. 38). From a practical point of view therefore it makes no difference whether this genus is regarded as being monotypical or as having its type species determined by subsequent selection, for on either view Hippolyte varians Leach is the type species.

(8) Notostomus Milne Edwards (A.), 1881 : In a footnote referring to the name Notostomus, Milne Edwards (A.) stated in the original description -of the genus: "de $\nu \grave{\omega} \tau o s$ dos et $\sigma \tau o ́ \mu \alpha$, le coupant d'un couteau". One would therefore be inclined to consider the spelling "Notostoma" (neuter) the correct one for the present name. However, Milne Edwards used the name Notostomus three times with this spelling in his 1881 paper and treated it as a masculine word, and in this he has been followed by all subsequent authors. Under the provisions adopted by the Copenhagen Congress, relating to the emendation of names, the 
spelling adopted for this name by Milne Edwards is not subject to emendation, except by the use by the Commission of its Plenary Powers, and in view of the general acceptance of the original spelling in this case, I do not recommend that course. I accordingly propose that this generic name should be placed on the Official List in its original spelling and that, in taking this decision, the Commission should direct that this generic name is to be treated as being masculine in gender.

(9) Oplophorus Milne Edwards (H.), 1837: Agassiz, 1846 (Nomencl. Zool., Index Univ. : 185) and several later authors have emended this name to Hoplophorus but since Milne Edwards in the original publication consistently used the name Oplophorus and said nothing about the derivation of this name, this emendation is, in my opinion, clearly invalid. The Commission is therefore asked to place this name on the Official List of Generic Names in Zoology in the original spelling Oplophorus, at the same time placing the emended spelling Hoplophorus on the Official Index of Rejected and Invalid Generic Names in Zoology.

(10) eugonatonotidae Chace, 1937 : This family name possesses a senior objective synonym, viz., GOMPHONOTIDAE Chace, 1936, as the type genera of these two families, Eugonatonotus Schmitt, 1926, and Gomphonotus Chace, 1936, have the same type species, Gonatonotus crassus Milne Edwards (A.), 1881. In my opinion, it is desirable to use the junior family name EUGONATONOTIDAE as this is based on the valid generic family name Eugonatonotus Schmitt, and to reject the older family name GOMPHONOTIDAE which is based on the name Gomphonotus Chace which is an invalid objective junior synonym of Eugonatonotus. The Commission is accordingly asked to use its Plenary Powers for this purpose and, having done so, to place the name EUGONATONOTIDAE on the Official List and that of GOMPHONOTIDAE on the Official Index of Rejected and Invalid FamilyGroup Names in Zoology.

(11) hippolytidae Bate, 1888: This family name has been accepted by all modern carcinologists. However, two of the genera now currently placed in the family HIPPOLYTIDAE are the type genera of Family-, Group names, which are older than the name HIPPOLYTIDAE. The Family-Group Names concerned are :-

LYSmatinae Dana, 1852, Proc. Acad. nat. Sci. Philad. 6 : 16, 20 (type genus: Lysmata Risso, 1816);

THORINAE Kingsley, 1878, Bull. Essex Inst. 10 : 64 (type genus : Thor Kingsley, 1878).

These two names have been ignored by all modern carcinologists. The stability of carcinological nomenclature would be greatly furthered if the name HIPPOLYTIDAE be given preference over the 
names LYSMATINAE and THORINAE. For this reason $I$ ask the International Commission to direct under its Plenary Powers that the family-group name HIPPOLYTIDAE Bate, 1888, is not to be rejected (a) in favour of the name Lysmatinae Dana, 1852, by any worker who on taxonomic grounds places the genus Lysmata Risso, 1816, in the same family-group taxon as Hippolyte Leach, 1814, or (b) in favour of the name THORINAE Kingsley, 1878, by any worker who on similar grounds places the genus Thor Kingsley, 1878, in the same familygroup taxon as Hippolyte Leach, 1814. The Commission is further asked to place the family-group name HIPPOLYTIDAE Bate, 1888, on the Official List of Family-Group Names in Zoology with a note in the foregoing terms.

3. The following list contains the required particulars regarding the one hundred and two generic names which it is now recommended should be placed on the Official List of Generic Names in Zoology :-

\section{Names recommended for addition to the " Official List of Generic Names in Zoology"}

Alope (feminine) White, 1847, Proc. zool. Soc. Lond. $15: 123$ (type species, by monotypy: Alope palpalis White, 1847, Proc. zool. Soc. Lond. $15: 124$ [Note (not for inclusion in the Official List) : This name is a junior subjective synonym of Hippolyte spinifrons Milne Edwards (H.), 1837, Hist. nat. Crust. 2 : 377]).

Anchistioides (masculine) Paulson, 1875, Issljed Rakoobr. Krasn. Morja (Stud. Crust. Red Sea) : 115 (type species, by monotypy: Anchistioides compressus Paulson, 1875, Issljed. Rakoobr. Krasn. Morja (Stud. Crust. Red Sea) : 115).

Anchistus (masculine) Borradaile, 1898, Ann. Mag. nat. Hist. (7) $2: 387$ (type species, by original designation: Harpilius miersi De Man, 1888, J. linn. Soc. Lond. (Zool.) 22 : 274).

Arete (feminine) Stimpson, 1860, Proc. Acad. nat. Sci. Philad. $1860: 32$ (type species, by monotypy: Arete dorsalis Stimpson, 1860, Proc. Acad. nat. Sci. Philad. $1860: 32$ ).

Argis (feminine) Kröyer, 1842, Naturhist. Tidsskr. 4(3) : 255, 267 (type species, by monotypy : Crangon lar Owen, 1839, Zool. Beechey's Voy. Blossom : 88).

Athanas (masculine) Leach, 1814, Brewster's Edinb. Encycl. 7(2) : 432 (type species, by monotypy: Palaemon nitescens Leach, 1814, Brewster's Edinb. Encycl. 7(2) : 401).

Atya (feminine) Leach, 1816, Encycl. Brit., Suppl. to 4th-6th eds. $1: 421$ (type species, by monotypy: Atys scaber Leach, 1815, Trans. linn. Soc. Lond. 11 : 345). 
Atyella (feminine) Calman, 1906, Proc. zool. Soc. Lond. 1906(1) : 201 (type species, by original designation: Atyella brevirostris Calman, 1906, Proc. zool. Soc. Lond. 1906(1) : 201).

Automate (feminine) De Man, 1888, Arch. Naturgesch. 53(1) : 529 (type species, by monotypy: Automate dolichognatha De Man, 1888, Arch. Naturgesch. $53(1): 529)$.

Balssia (feminine) Kemp, 1922, Rec. Indian Mus. 24 : 267 (type species, by monotypy: Amphipalaemon gasti Balss, 1921, Mitt. zool. Sta. Neapel $22: 523)$.

Barbouria (feminine) Rathbun, 1912, Bull. Mus. comp. zool. 54 : 455 (type species, by monotypy: Barbouria poeyi Rathbun, 1912, Bull. Mus. comp. zool. $54: 455$ ) [Note (not for inclusion in the Official List): This name is a junior subjective synomym of Hippolyte cubensis Von Martens, 1872, Arch. Naturgesch. 38(1) : 136]).

Bathypalaemonella (feminine) Balss, 1914, Zool. Anz. 44 : 597 (type species, by monotypy: Bathypalaemonella zimmeri Balss, 1914, Zool. Anz. 44 : 598).

Betaeus (masculine) Dana, 1852, Proc. Acad. nat. Sci. Philad. 6 : 16 (type species, by selection by Fowler, 1912 (Ann. Rep. New Jersey State Mus. 1911 : 558) : Betaeus truncatus Dana, 1852, Proc. Acad. nat. Sci. Philad. $6: 23)$.

Birulia (feminine) Brashnikov, 1903, Annu. Mus. zool. Petersb. 8 : xliv (type species, by monotypy: Birulia sachalinensis Brashnikov, 1903, Annu. Mus. zool. Petersb. 8 : xlv).

Brachycarpus (masculine) Bate, 1888, Rep. Voy. Challenger, Zool. 24 : 781, 795 (type species, by original designation: Brachycarpus savignyi Bate, 1888, Rep. Voy. Challenger, Zool. 24 : 795 [Note (not for inclusion in the Official List): This name is a junior subjective synonym of Palaemon biunguiculatus Lucas, 1849, Explor. sci. Algérie, Hist. nat., Anim. art. (1) : 45]).

Bresilia (feminine) Calman, 1896, Trans. Roy. Irish Acad. $31: 7$ (type species, by monotypy: Bresilia atlantica Calman, 1896, Trans. Roy. Irish Acad. $31: 7)$.

Bythocaris (feminine) Sars (G. O.), 1870, Forh. Vidensk. Selsk. Christiania 1869 : 149 (type species, by monotypy: Bythocaris simplicirostris Sars (G.O.), 1870, Forh. Vidensk. Selsk. Christiania 1869 : 149).

Campylonotus (masculine) Bate, 1888, Rep. Voy. Challenger, Zool, 24 : 767 (type species, by selection by Holthuis, 1955 (Zool. Verhand. Leiden $26: 41$ ) : Campylonotus semistriatus Bate, 1888, Rep. Voy. Challenger, Zool. 24 : 768). 
Caridella (feminine) Calman, 1906, Proc. zool. Soc. Lond. 1906(1) : 198 (type species, by original designation: Caridella cunningtoni Calman, 1906, Proc. zool. Soc. Lond. 1906(1) : 199).

Caridina (feminine) Milne Edwards (H.), 1837, Hist. nat. Crust. 2 : 362 (type species, by indication under Rule (b) in Article 30: Caridina typus Milne Edwards (H.), 1837, Hist. nat. Crust. 2 : 363).

Caridion (masculine) Goës, 1863, Ofvers. K. Svensk. Vetensk. Akad. Förh. 20 : 170 (substitute name for Doryphorus Norman, 1861 (Ann. Mag. nat. Hist. (3)8 : 276, an invalid junior homonym of Doryphorus Cuvier, 1829, Règne Anim. (ed. 2) 2 : 34) (type species, by monotypy for Doryphorus Norman, 1861 : Hippolyte gordoni Bate, 1858, Nat. Hist. Rev. Proc. Soc. Dublin 5 : iv).

Chlorocurtis (masculine) Kemp, 1925, Rec. Indian Mus. $27: 272,279$ (type species, by monotypy: Chlorocurtis miser Kemp, 1925, Rec. Indian Mus. $27: 280$ [Note (not for inclusion in the Official List) : This name is a junior subjective synonym of Virbius (?) jactans Nobili, 1904, Bull. Mus. Hist. nat. Paris $10: 230])$.

Chlorotocella (feminine) Balss, 1914, Abh. Bayer. Akad. Wiss. Suppl. 2(10) : 33 (type species, by monotypy : Chlorotocella gracilis Balss, 1914, Abh. Bayer. Akad. Wiss. Suppl. 2(10) : 33).

Chlorotocoides (masculine) Kemp, 1925, Rec. Indian Mus. 27 : 271, 276 (type species, by monotypy: Chlorotocus spinicauda De Man, 1902, Abh. Senckenb. naturf. Ges. $25: 856$ ).

Chlorotocus (masculine) Milne Edwards (A.), 1882, Arch. Miss. sci. litt. (3)9 : 14 (type species, by monotypy: Chlorotocus gracilipes Milne Edwards (A.), 1882, Arch. Miss. sci. litt. (3)9: 14 [Note (not for inclusion in the Official List) : This name is a junior subjective synonym of Pandalus crassicornis Costa, 1871, Annu. Mus. zool. Univ. Napoli 6 : 89]).

Chorismus (masculine) Bate, 1888, Rep. Voy. Challenger, Zool. 24 : 577, 616 (type species, by selection by Calman, 1907 (Nat. Antarct. Exped., Nat. Hist. 2 (Crust.)(1) : 3) : Chorismus tuberculatus Bate, 1888, Rep. Voy. Challenger, Zool. $24: 617$ ).

Coralliocaris (feminine) Stimpson, 1860, Proc. Acad. nat. Sci. Philad. 1860 : 38 (substitute name for Oedipus Dana, 1852, Proc. Acad. nat. Sci. Philad. $6: 17$, an invalid junior homonym of Oedipus Berthold, 1827, in Latreille, Nat. Fam. Thierr. : 411) (type species, by selection by Kingsley, 1880 (Proc. Acad. nat. Sci. Philad. 1879 : 423) : Oedipus superbus Dana, 1852, Proc. Acad. nat. Sci. Philad. 6 : 25).

Coutierea (feminine) Nobili, 1901, Boll. Mus. Zool. Anat. comp. Torino 16(415) : 4 (type species, by monotypy: Coralliocaris agassizi Coutière, 1901, Bull. Mus. Hist. nat., Paris 7 : 115). 
Cryptocheles (feminine) Sars (G. O.), 1870. Forh. Vidensk. Selsk. Christiania 1869 : 150 (type species, by monotypy : Cryptocheles pygmaea Sars (G. O.), 1870, Forh. Vidensk. Selsk. Christiania 1869 : 150).

Dasycaris (feminine) Kemp, 1922, Rec. Indian Mus. $24: 240$ (type species, by monotypy: Dasycaris symbiotes Kemp, 1922, Rec. Indian Mus. 24: 240).

Desmocaris (feminine) Sollaud, 1911, C. R. Acad. Sci., Paris 152: 913 (type species, by monotypy: Palaemonetes trispinosus Aurivillius, 1898, Bih. Svenska Vetensk. Akad. Handl. 24(4)(1) : 29).

Dichelopandalus (masculine) Caullery, 1896, Ann. Univ. Lyon $26: 379$ (type species, by monotypy: Dichelopandalus bonnieri Caullery, 1896, Ann. Univ. Lyon $26: 379$ ).

Dorodotes (masculine) Bate, 1888, Rep. Voy. Challenger, Zool. 24:627, 677 (type species, by selection by Holthuis, 1955 (Zool. Verhand. Leiden $26: 119$ ) : Dorodotes reflexus Bate, 1888, Rep. Voy. Challenger, Zool. 24 : 678).

Dugastella (feminine) Bouvier, 1912, C. R. Acad. Sci., Paris 155 : 993 (type species, by monotypy: Dugastella marocana Bouvier, 1912, C. R. Acad. Sci., Paris $155: 993)$.

Ephyrina (feminine) Smith (S. I.), 1885, Proc. U.S. Nat. Mus. 7 : 506 (type species, by monotypy: Ephyrina benedicti Smith (S. I.), 1885, Proc. U.S. nat. Mus. 7 : 506).

Eugonatonotus (masculine) Schmitt, 1926, Biol. Res. Fish. Exper. "Endeavour" 5(6) : "Corringenda et Addenda" sheet, second page (substitute name for Gonatonotus Milne Edwards (A.), 1881 (Ann. Sci.nat., Zool. (6)11(4) : 10, an invalid junior homonym of Gonatonotus Adams \& White, 1847) (type species, by monotypy for Gonatonotus Milne Edwards (A.), 1881 : Gonatonotus crassus Milne Edwards (A.), 1881, Ann. Sci. nat., Zool. (6)11(4) : $10)$.

Gelastocaris (feminine) Kemp, 1914, Rec. Indian Mus. $10: 106$ (type species, by monotypy: Latreutes paronae Nobili, 1905, Boll. Mus. Zool. Anat. comp. Torino 20(506): 2).

Glyphocrangon (feminine) Milne Edwards (A.), 1881, Ann. Sci. nat., Zool. (6)11(4) : 3 (type species, by original designation: Glyphocrangon spinicauda Milne Edwards (A.), 1881, Ann. Sci. nat., Zool. (6)11(4) : 3).

Gnathophyllum (neuter) Latreille, 1819, Nouv. Dict. Hist. nat. (ed. 2) $30: 72$ (type species, by selection by Milne Edwards (H.), 1837 (Cuvier's Règne Anim. (ed. 4, Disciples' ed.) 18 : pl. 52 fig. 2) : Alpheus elegans Risso, 1816, Hist. nat. Crust. Nice : 92). 
Heptacarpus (masculine) Holmes, 1900, Occ. Pap. Calif. Acad. Sci. 7 : 195 (type species, by original designation: Hippolyte palpator Owen, 1839, Zool. Beechey's Voy. Blossom : 89).

Heterocarpoides (masculine) De Man, 1917, Zool. Meded. Leiden 3 : 284 (type species, by monotypy: Dorodotes levicarina Bate, 1888, Rep. Voy. Challenger, Zool. 24 : 680).

Heterocarpus (masculine) Milne Edwards (A.), 1881, Ann. Sci. nat., Zool. (6)11(4) : 8 (type species, by original designation: Heterocarpus ensifer Milne Edwards (A.), 1881, Ann. Sci. nat. Zool. (6)11(4) : 8).

Hippolysmata (feminine) Stimpson, 1860, Proc. Acad.nat. Sci. Philad. 1860 : 26 (type species, by monotypy: Hippolysmata vittata Stimpson, 1860, Proc. Acad. nat. Sci. Philad. 1860 : 26).

Hippolyte (feminine) Leach, 1814, Brewster's Edinb. Ency. 7(2):431 (type species, by subsequent selection by Leach, 1817 (Malac. podophth. Brit. (16) : first page of expl. to pl. 38): Hippolyte varians Leach, 1814, Brewster's Edinb. Ency. 7(2) : 431).

Hymenodora (feminine) Sars (G. O.), 1877, Arch. Math. Naturvidensk. 2 : 340 [240] (type species, by monotypy: Pasiphaea glacialis Buchholz, 1874, Zweite Deutsche Nordpolarfahrt $2: 279$ ).

Latreutes (masculine) Stimpson, 1860, Proc. Acad. nat. Sci. Philad. 1860 : 27 (type species, by selection by Kingsley, 1880 (Proc. Acad. nat. Sci. Philad. 1879 : 413): Hippolyte ensiferus Milne Edwards (H)., 1837, Hist. nat. Crust. $2: 374$ [Note (not for inclusion in the Official List): This name is a junior subjective synonym of Palaemon fucorum Fabricius, 1798, Suppl. Ent. Syst. : 404]).

Leontocaris (feminine) Stebbing, 1905, Mar. Invest. S. Afr. $4: 21,98$ (type species, by monotypy: Leontocaris paulsoni Stebbing, 1905, Mar. Invest. S. Afr. $4: 99)$.

Leptochela (feminine) Stimpson, 1860, Proc. Acad. nat. Sci. Philad. $1860: 42$ (type species, by selection by Holthuis, 1955 (Zool. Verhand. Leiden $26: 36$ ) : Leptochela gracilis Stimpson, 1860, Proc. Acad. nat. Sci. Philad. 1860 : 42).

Ligur (masculine) Sarato, 1885, Moniteur des Etrangers Nice 9(222) : 2 (type species, by monotypy : Ligur edwardsii Sarato, 1885, Moniteur des Etrangers Nice $9(222): 2$ [Note (not for inclusion in the Official List) : This name is a junior subjective synonym of Palemon ensiferus Risso, 1816, Hist. nat. Crust. Nice : 106]).

Limnocaridella (feminine) Bouvier, 1913, Bull. Soc. ent. France 1913 : 180 (type species, by monotypy: Limnocaridina alberti Lenz, 1910, Wiss. Ergebn. Deutsch. Zentral-Afr. Exped. 1907-1908 3(3) : 12). 
Limnocaridina (feminine) Calman, 1899, Proc. zool. Soc. Lond. 1899 : 704 (type species, by monotypy: Limnocaridina tanganyikae Calman, 1899, Proc. zool. Soc. Lond. 1899 : 704).

Merguia (feminine) Kemp, 1914, Rec. Indian Mus. 10:121 (type species, by monotypy : Hippolyte oligodon De Man, 1888, J. linn. Soc. Lond., Zool. 22 : 277).

Merhippolyte (feminine) Bate, 1888, Rep. Voy. Challenger, Zool. 24 : 577, 618 (type species by original designation : Merhippolyte agulhasensis Bate, 1888, Rep. Voy. Challenger, Zool. 24 : 619).

Micratya (feminine) Bouvier, 1913, Bull. Soc. ent. France 1913 : 181 (type species, by monotypy: Atya poeyi Guérin-Ménéville, 1856, in Sagra (R. de la), Hist. Cuba, Hist. nat. 7 : xviii).

Mimocaris (feminine) Nobili, 1903, Boll. Mus. Zool. Anat. comp. Torino 18(447) : 5 (type species, by monotypy : Mimocaris heterocarpoides Nobili, 1903, Boll. Mus. Zool. Anat. comp. Torino 18(447) : 6).

Nauticaris (feminine) Bate, 1888, Rep. Voy. Challenger, Zool. 24:577, 602 (type species, by selection by Calman, 1906 (Ann. Mag. nat. Hist. (7)17 : 31): Nauticaris marionis Bate, 1888, Rep. Voy. Challenger, Zool. 24 : 603).

Nematocarcinus (masculine) Milne Edwards (A.), 1881, Ann. Sci. nat., Zool. (6)11(4) : 14 (type species, by monotypy: Nematocarcinus cursor Milne Edwards (A.), 1881, Ann. Sci. nat., Zool. (6)11(4) : 14).

Nikoides (masculine) Paulson, 1875, Issljed. Rakoobr. Krasn. Morja (Stud. Crust. Red Sea) : 98 (type species, by monotypy : Nikoides danae Paulson, 1875, Issljed. Rakoobr. Krasn. Morja (Stud. Crust. Red Sea) : 98).

Notocrangon (feminine) Coutière, 1900, C. R. Acad. Sci., Paris, $130: 1640$ (type species, by monotypy: Crangon antareticus Pfeffer, 1887, Jahrb., Hamb. wiss. Anst. $4: 45)$.

Notostomus (masculine) Milne Edwards (A.), 1881, Ann. Sci. nat., Zool. (6)11(4) :7 (type species, by original designation: Notostomus gibbosus Milne Edwards (A.), 1881, Ann. Sci. nat., Zool. (6)11(4) : 7).

Ogyrides (masculine) Stebbing, 1914, Ann. S. Afr. Mus. $15: 31$ (substitute name for Ogyris Stimpson, 1860, Proc. Acad.nat. Sci. Philad. 1860 : 36, an invalid junior homonym of Ogyris Westwood, [1851], in Doubleday, Gen. diurn. Lep. (2) : pl. 75, fig. 8) (type species, by monotypy for Ogyris Stimpson, 1860: Ogyris orientalis Stimpson, 1860, Proc. Acad. nat. Sci. Philad. $1860: 36)$. 
Oplophorus (masculine) Milne Edwards (H.), 1837, Hist. nat. Crust. 2 : 423 (type species, by indication under Rule (b) in Article 30: Oplophorus typus Milne Edwards (H.), 1837, Hist. nat. Crust. 2 : 424).

Palaemonella (feminine) Dana, 1852, Proc. Acad. nat. Sci. Philad. 6 : 17 (type species, by selection by Kingsley, 1880 (Proc. Acad. nat. Sci. Philad. 1879 : 425): Palaemonella tenuipes Dana, 1852, Proc. Acad. nat. Sci. Philad. 6 : 25).

Palaemonetes (masculine) Heller, 1869, Z. wiss. zool. 19 : 157, 161 (type species, by monotypy: Palaemon varians Leach, 1814, Brewster's Edinb. Ency. $7(2): 432)$.

Palaemonias (masculine) Hay, 1901, Proc. biol. Soc. Wash. 14: 179 (type species, by monotypy: Palaemonias ganteri Hay, 1901, Proc. biol. Soc. Wash. $14: 180$ ).

Pandalina (feminine) Calman, 1899, Ann. Mag. nat. Hist. (7)3 : 37 (type species, by monotypy : Pandalus brevirostris Rathke, 1843, Nova Acta Acad. Leop. Carol. 20(1) : 17).

Pantomus (masculine) Milne Edwards (A.), 1883, Rec. Fig. Crust. nouv. peu conn. : pl. 26, fig. 1 (type species, by monotypy: Pantomus parvulus Milne Edwards (A.), 1883, Rec. Fig. Crust. nouv. peu conn. : pl. 26 fig. 1).

Paracrangon (feminine) Dana, 1852, Proc. Acad. nat. Sci. Philad. 6 : 16, 20 (type species, by monotypy: Paracrangon echinatus Dana, 1852, Proc. Acad. nat. Sci. Philad. $6: 20$ ).

Paralatreutes (masculine) Kemp, 1925, Rec. Indian Mus. 27 : 334 (type species, by monotypy: Paralatreutes bicornis Kemp, 1925, Rec. Indian Mus. $27: 334)$.

Parapandalus (masculine) Borradaile, 1899, Willey's Zool. Res. (4) : 411 (type species, by selection by Alcock, 1901 (Descr. Catal. Indian Deep-Sea Crust. Decap. Macr. Anom. : 94) : Pandalus (Parapandalus) serratifrons Borradaile, 1899, Willey's Zool. Res. (4) : 411 [Note (not for inclusion in the Official List): This name is a junior subjective synonym of Plesionika spinipes Bate, 1888, Rep. Voy. Challenger, Zool. 24 : 646]).

Parapasiphaë (feminine) Smith (S. I.), 1884, Rep. U.S. Fish Comm. $10: 383$ (type species, by selection by Fowler, 1912 (Ann. Rep. New Jersey State Mus. $1911: 547$ ) : Parapasiphaë suleatifrons Smith (S. I.), 1884, Rep. U.S. Fish Comm. $10: 384)$.

Paratya (feminine) Miers, 1882, Ann. Mag. nat. Hist. (5)9 : 194 (type species, by monotypy: Ephyra compressa De Haan, 1844, Fauna japon., Crust. $(6 / 7)$ : pl. 46 fig. 7$)$.

Paratypton (masculine) Balss, 1914, Zool. Anz. 45 : 83 (type species, by monotypy : Paratypton siebenrocki Balss, 1914, Zool. Anz. 45 : 84). 
Pasiphaea (feminine) Savigny, 1816, Mém. Anim. sans Vertèbr. 1 : 50 (type species, by monotypy:: Alpheus sivado Risso, 1816, Hist. nat. Crust. Nice : 93).

Periclimenaeus (masculine) Borradaile, 1915, Ann. Mag. nat. Hist. (8)15 : 207 (type species, by selection by Borradaile, 1917 (Trans. linn. Soc. Lond., Zool. (2)17 : 378) : Periclimenaeus robustus Borradaile, 1915, Ann. Mag. nat. Hist. (8)15 : 213).

Periclimenes (masculine) Costa, 1844, Ann. Accad. Aspir. Nat. Napoli 2 : 290 (type species, by monotypy: Periclimenes insignis Costa, 1844, Ann. Accad. Aspir. Nat. Napoli 2 : 291 [Note (not for inclusion in the Official List) : This name is a junior subjective synonym of Alpheus amethystea Risso, 1826, Hist. nat. Europ. mérid. 5 : 77]).

Phycocaris (feminine) Kemp, 1916, Rec. Indian Mus. 12 : 391 (type species, by monotypy: Phycocaris simulans Kemp, 1916, Rec. Indian Mus. 12 : 392).

Phyllognathia (feminine) Borradaile, 1915, Ann. Mag. nat. Hist. (8)15 : 205, 206 (type species, by monotypy: Hymenocera (?) ceratophthalma Balss, 1913, Zool. Anz. 42 : 236).

Plesionika (feminine) Bate, 1888, Rep. Voy. Challenger, Zool. 24 : 626, 640 (type species, by selection by Alcock, 1901 (Descr. Catal. Indian Deep-Sea Crust. Decap. Macr. Anom. : 93) : Plesionika uniproducta Bate, 1888, Rep. Voy. Challenger, Zool. 24:641 [Note (not for inclusion in the Official List): This name is a junior subjective synonym of Acanthephyra ensis Milne Edwards (A.), 1881, Ann. Sci. nat., Zool. (6)11(4) : 14]).

Pontocaris (feminine) Bate, 1888, Rep. Voy. Challenger, Zool. 24: 495 (type species, by selection by Holthuis, 1947 (Zool. Meded. $27: 320)$ : Pontocaris propensalata Bate, 1888, Rep. Voy. Challenger, Zool. 24 : 496).

Pontonides (masculine) Borradaile, 1917, Trans. linn. Soc. Lond., Zool. (2)17 : 387 (type species, by monotypy: Pontonia maldivensis Borradaile, 1915, Ann. Mag. nat. Hist. (8)15: 213).

Prionocrangon (feminine) Wood Mason \& Alcock, 1891, Ann. Mag. nat. Hist. (6) $8: 361$ (type species, by monotypy: Prionocrangon ommatosteres Wood Mason \& Alcock, 1891, Ann. Mag. nat. Hist. (6)8 : 362).

Psalidopus (masculine) Wood Mason \& Alcock, 1892, Ann. Mag. nat. Hist. (6)9 : 266 (type species, by selection by Holthuis, 1955 (Zool. Verhand. Leiden $26: 81)$ : Psalidopus huxleyi Wood Mason \& Alcock, 1892, Ann. Mag. nat. Hist. (6)9 : 273).

Psathyrocaris (feminine) Wood Mason \& Alcock, 1893, Ann. Mag. nat. Hist. (6)11: 168 (type species, by monotypy: Psathyrocaris fragilis Wood Mason \& Alcock, 1893, Ann. Mag. nat. Hist. (6)11 : 168).

Pseudopalaemon (masculine) Sollaud, 1911, Bull. Mus. Hist. nat., Paris 17 : 12, 15 (type species, by monotypy: Pseudopalaemon bouvieri Sollaud, 1911, Bull. Mus. Hist. nat., Paris 17 : 12). 
Rhynchocinetes (masculine) Milne Edwards (H.), 1837, Ann. Sci. nat., Zool. (2)7 : 168 (type species, by indication under Rule (b) in Article 30 : Rhynchocinetes typus Milne Edwards (H.), 1837, Ann. Sci. nat., Zool. $(2) 7: 168)$.

Sabinea (feminine) Ross (J. C.), 1835, J. Ross's App. Narrat. 2nd Voy. N.W. Passage : lxxxii (type species, by monotypy: Crangon septemcarinatus Sabine, 1824, Suppl. App. Parry's Voy. N.W. Passage : cexxxvi).

Saron (masculine) Thallwitz, 1891, Zool. Anz. 14 : 99 (type species, by monotypy: Hippolyte gibberosus Milne Edwards (H.), 1837, Hist. nat. Crust. 2 : 378 [Note (not for inclusion in the Official List): This name is a junior subjective synonym of Palaemon marmoratus Olivier, 1811, Ency. méth. Hist. nat. $8: 663])$.

Sclerocrangon (feminine) Sars (G. O.), 1883 (sep. 1882), Forh. Vidensk. Selsk. Christiania 1882(18) : 7, 45 (type species, by monotypy: Cancer boreas Phipps, 1774, Voy. North Pole : 190).

Stegopontonia (feminine) Nobili, 1906, Bull. Mus. Hist. nat., Paris, 12 : 258 (type species, by monotypy: Stegopontonia commensalis Nobili, 1906, Bull. Mus. Hist. nat., Paris 12 : 258).

Stylodactylus (masculine) Milne Edwards (A.), 1881, Ann. Sci. nat., Zool. (6)11(4) : 11 (type species, by monotypy: Stylodactylus serratus Milne Edwards (A.), 1881, Ann. Sci. nat., Zool. (6)11(4) : 11).

Synalpheus (masculine) Bate, 1888, Rep. Voy. Challenger, Zool. 24 : 572 (type species, by monotypy : Synalpheus falcatus Bate, 1888, Rep. Voy. Challenger, Zool. 24: 574 [Note (not for inclusion in the Official List): This name is a junior subjective synonym of Alpheus comatularum Haswell, 1882, Proc. linn. Soc. New S. Wales 6 : 762]).

Syncaris (feminine) Holmes, 1900, Occ. Pap. Calif. Acad. Sci. 7 : 211 (type species, by original designation: Miersia pacifica Holmes, 1895, Proc. Calif. Acad. Sci. (2)4: 577).

Systellaspis (feminine) Bate, 1888, Rep. Voy. Challenger, Zool. 24 : 757 (type species, by original designation: Systellaspis lanceocaudata Bate, 1888, Rep. Voy. Challenger, Zool. 24 : 758).

Thalassocaris (feminine) Stimpson, 1860, Proc. Acad. nat. Sci. Philad. $1860: 42$ (substitute name for Regulus Dana, 1852, Proc. Acad.nat. Sci. Philad. 6 : 18, a junior homonym of Regulus Cuvier (G.L.C.F.D.), 1800, Lecons Anat. comp. 1 : Tab. 2) (type species, by selection by Kingsley, 1880, Proc. Acad. nat. Sci. Philad. 1879 : 426) : Regulus lucidus Dana, 1852, Proc. Acad. nat. Sci. Philad. 6 : 27). 
Thaumastocaris (feminine) Kemp, 1922, Rec. Indian Mus. $24: 244$ (type species, by monotypy: Thaumastocaris streptopus Kemp, 1922, Rec. Indian Mus. 24 : 244).

Thor (masculine) Kingsley, 1878, Proc. Acad. nat. Sci. Philad. 1878 : 94 (type species, by monotypy: Thor floridanus Kingsley, 1878, Proc. Acad. nat. Sci. Philad. 1878 : 95).

Trachycaris (feminine) Calman, 1906, Ann. Mag. nat. Hist. (7)17 : 31, 33 (type species, by monotypy: Platybema rugosus Bate, 1888, Rep. Voy. Challenger, Zool. 24:579 [Note (not for inclusion in the Official List): This name is a junior subjective synonym of Hippolyte restrictus Milne Edwards (A.), 1878, Bull. Soc. philom. Paris (7)2 : 231]).

Troglocaris (feminine) Dormitzer, 1853, Lotos $3: 85$ (type species, by monotypy: Troglocaris schmidti Dormitzer, 1853, Lotos $3: 85$ [Note (not for inclusion in the Official List) : This name is a junior subjective synonym of Palaemon anophthalmus Kollar, 1848, S. B. Akad. Wiss. Wien 1 : 137]).

Typhlocaris (feminine) Calman, 1909, Trans. linn. Soc. Lond., Zool. (2)11 : 93, 94 (type species, by monotypy: Typhlocaris galilea Calman, 1909, Trans. linn. Soc. Lond., Zool. (2)11 : 93).

Typton (masculine) Costa, 1844, Ann. Accad. Aspir. Nat. Napoli 2 : 288 (type species, by monotypy: Typton spongicola Costa, 1844, Ann. Accad. Aspir. Nat. Napoli 2 : 289).

Xiphocaris (feminine) Von Martens, 1872, Arch. Naturgesch. 38(1) : 139 (type species, by monotypy: Hippolyte elongatus Guérin-Ménéville, 1856, in Sagra (R. de la), Historia Cuba, Hist. nat. 7 : xx).

4. At the time when I submitted the present application to the Commission, I included in it proposals for the addition to the Official List of the names of four other genera, each of which has the termination " -opsis". These names were : (1) Alpheopsis Coutière, 1897; (2) Caridinopsis Bouvier, 1912 ; (3) Pandalopsis Bate, 1888 ; (4) Pontoniopsis Borradaile, 1915. These names, like other generic names with the same termination in the Order Decapoda, are invariably treated as being masculine in gender and I accordingly recommended that this gender should be attributed to them when placed on the Official List. Recently, Mr. Francis Hemming, as Secretary, has drawn my attention to the decision by the Fourteenth International Congress of Zoology, Copenhagen, 1953, that generic names having the foregoing termination are to be treated as being feminine in gender (1953, Copenhagen Decisions zool. Nomencl. : 51, Decision 84(7)(b)(iii)). Mr. Hemming has informed me also that the problem of the gender to be attributed to generic names in the Decapoda having this termination has arisen in regard to certain such names which were placed on the Official List of Generic Names in Zoology in the period prior to the Lisbon Congress and to which therefore a gender must now be assigned by the Commission. Mr. Hemming has further informed me 
that in view of the clash in this case between linguistic considerations and the general practice of carcinologists, it is his intention to take the directions of the Commission separately in regard to the gender to be assigned to the generic names referred to above. It is evident that, whatever decision is taken by the Commission on the foregoing names will need to apply also to the four names of this group which I had originally included in the present application. At Mr. Hemming's suggestion, I have therefore withdrawn from the present application my proposals in regard to the four names cited above. My proposals in regard to these names will be laid before the Commission in a separate application, to which, Mr. Hemming informs me, the Registered Number Z.N.(S.) 919 has been allotted.

5. It is recommended that the specific names of the type species of the genera specified in paragraph 3 above should be placed on the Official List of Specific Names in Zoology, in as far as these names are valid and at the same time are the oldest available names for the species concerned. The following list gives in the first column the specific names which fulfil the conditions mentioned above. In the second column is given the original combination in which these names have been used. In this column the spelling both of the specific and of the generic names is emended in accordance with the Règles as amended by the International Congress of Zoology, Copenhagen, 1953, and conform with the suggestions made in paragraph 2 of the present proposal. In column (3) is given the name of the genus of which the species cited in column (1) is the type species.

\section{Specific Name}

agassizi Coutière, 1901

agulhasensis Bate, 1888

alberti Lenz, 1910

antarcticus Pfeffer, 1887

atlantica Calman, 1896

benedicti Smith (S. I.), 1885

bicornis Kemp, 1925

bonnieri Caullery, 1896

boreas Phipps, 1774
Original Combination

in which name cited in

Col. (1) was published

Coralliocaris agassizi

Merhippolyte agulhasensis

Limnocaridina alberti

Crangon antarcticus

Bresilia atlantica

Ephyrina benedicti

Paralatreutes bicornis

Dichelopandalus

bonnieri

Cancer boreas
Genus of which species eited in Col. (1) is the type species

Coutierea Nobili, 1901

Merhippolyte Bate, 1888

Limnocaridella Bouvier, 1913

Notocrangon Coutière, 1900

Bresilia Calman, 1896

Ephyrina Smith (S. I.), 1885

Paralatreutes Kemp, 1925

Dichelopandalus Caullery, 1896

Sclerocrangon Sars (G. O.), 1883 


\section{Specific Name}

(1)

bouvieri Sollaud, 1911

brevirostris Rathke, 1843

brevirostris Calman, 1906

ceratophthalma Balss,

1913

commensalis Nobili, 1906

compressa De Haan, 1841

compressus Paulson, 1875

crassus Milne Edwards

(A.), 1881

cunningtoni Calman, 1906

cursor Milne Edwards

(A.), 1881

danae Paulson, 1875

dolichognatha De Man, 1888

dorsalis Stimpson, 1860

echinatus Dana, 1852

elegans Risso, 1816

elongatus Guérin-

Ménéville, 1856 ensifer Milne Edwards Heterocarpus ensifer
(A.), 1881

floridanus Kingsley, 1878 fragilis Wood Mason \& Alcock, 1893

galilea Calman, 1909

ganteri Hay, 1901

gasti Balss, 1921

gibbosus Milne Edwards

(A.), 1881
Original Combination in which name cited in Col. (1) was published

(2)

Pseudopalaemon bouvieri

Pandalus brevirostris

Atyella brevirostris

Hymenocera (?) ceratophthalma

Stegopontonia commensalis

Ephyra compressa

Anchistioides compressus

Gonatonotus crassus

Caridella cunningtoni

Nematocarcinus cursor

Nikoides danae

Automate

dolichognatha

Arete dorsalis

Paracrangon echinatus

Alpheus elegans

Hippolyte elongatus

Thor floridanus

Psathyrocaris fragilis

Typhlocaris galilea

Palaemonias ganteri

Amphipalaemon gasti

Notostomus gibbosus
Genus of which species cited in Col. (1) is the type species

Pseudopalaemon Sollaud, 1911

Pandalina Calman, 1899 Atyella Calman, 1906

Phyllognathia Borradaile, 1915

Stegopontonia Nobili, 1906

Paratya Miers, 1882

Anchistioides Paulson, 1875

Eugonatonotus Schmitt, 1926

Caridella Calman, 1906

Nematocarcinus Milne

Edwards (A.), 1881

Nikoides Paulson, 1875

Automate De Man, 1888

Arete Stimpson, 1860

Paracrangon Dana, 1852

Gnathophyllum Latreille, 1819

Xiphocaris Von Martens, 1872

Heterocarpus Milne

Edwards (A.), 1881

Thor Kingsley, 1878

Psathyrocaris Wood

Mason \& Alcock, 1893

Typhlocaris Calman, 1909

Palaemonias Hay, 1901

Balssia Kemp, 1922

Notostomus Milne

Edwards (A.), 1881 


\section{Specific Name}

Original Combination in which name cited in Col. (1) was published

\section{Genus of which species cited in Col. (1) is the type species}

glacialis Buchholz, 1874 Pasiphaë glacialis

gordoni Bate, 1858

gracilis Balss, 1914

gracilis Stimpson, 1860

Hippolyte gordoni

Leptochela gracilis
Chlorotocella gracilis

Mimocaris heterocarpoides 1903

huxleyi Wood Mason \& Psalidopus huxleyi Alcock, 1892

$\begin{array}{ll}\text { lanceocaudata Bate, } 1888 & \begin{array}{c}\text { Systellaspis } \\ \text { lanceocaudata }\end{array} \\ \text { lar Owen, } 1839 & \text { Crangon lar } \\ \text { levicarina Bate, } 1888 & \text { Dorodotes levicarina } \\ \text { lucidus Dana, } 1852 & \text { Regulus lucidus }\end{array}$

maldivensis Borradaile, 1915

marionis Bate, 1888

marocana Bouvier, 1912

miersi De Man, 1888

nitescens Leach, 1814

oligodon De Man, 1888

ommatosteres Wood

Mason \& Alcock, 1891

orientalis Stimpson, 1860

pacifica Holmes, 1895

palpator Owen, 1839

paronai (emend. of

paronae) Nobili, 1905

parvulus Milne Edwards

(A.), 1883
Pontonia maldivensis

Nauticaris marionis

Dugastella marocana

Harpilius miersi

Palaemon nitescens

Hippolyte oligodon

Prionocrangon

ommatosteres

Ogyris orientalis

Miersia pacifica

Hippolyte palpator

Latreutes paronai

Pantomus parvulus
(3)

Hymenodora Sars (G. O.), 1877

Caridion Goës, 1863

Chlorotocella Balss, 1914

Leptochela Stimpson, 1860

Mimocaris Nobili, 1903

Psalidopus Wood Mason \& Alcock, 1892

Systellaspis Bate, 1888

Argis Kröyer, 1842

Heterocarpoides De Man, 1917

Thalassocaris Stimpson, 1860

Pontonides Borradaile, 1917

Nauticaris Bate, 1888

Dugastella Bouvier, 1912

Anchistus Borradaile, 1898

Athanas Leach, 1814

Merguia Kemp, 1914

Prionocrangon Wood

Mason \& Alcock, 1891

Ogyrides Stebbing, 1914

Syncaris Holmes, 1900

Heptacarpus Holmes, 1900

Gelastocaris Kemp, 1914

Pantomus Milne

Edwards (A.), 1883 


\section{Specific Name}

(1)
Original Combination Col. (1) was published

paulsoni Stebbing, 1905 1856

propensalata Bate, 1888

pygmaea Sars (G. O.),1870

reflexus Bate, 1888

robustus Borradaile, 1915

Leontocaris paulsoni

Atya poeyi

Pontocaris propensalata in which name cited in

poeyi Guérin-Ménéville,
Dorodotes reflexus

Periclimenaeus robustus
Genus of which species cited in Col. (1) is the type species Cryptocheles pygmaea

Pontocaris Bate, 1888

Cryptocheles Sars (G. O.), 1870

Dorodotes Bate, 1888

Periclimenaeus

Borradaile, 1915

Birulia Brashnikov, 1903

Atya Leach, 1816

Campylonotus Bate, 1888

Campylonotus

semistriatus

Crangon septemcarinatus

Stylodactylus serratus

Paratypton siebenrocki

Bythocaris

simplicirostris

Phycocaris simulans

Alpheus sivado

Glyphocrangon

spinicauda

Chlorotocus spinicaudus

1902

spongicola Costa, 1844

streptopus Kemp, 1922

sulcatifrons Smith (S. I.),

1884

superbus Dana, 1852

symbiotes Kemp, 1922

$$
\text { tan }
$$$$
\begin{aligned}
& \text { s } \\
& \text { s } \\
& \text { sp } \\
& \text { sp } \\
& \text { sp } \\
& \text { sp } \\
& \text { si } \\
& \text { su } \\
& \text { sup } \\
& \text { sy }
\end{aligned}
$$ 


\section{Specific Name}

(1)
Original Combination in which name cited in Col. (1) was published

trispinosus Aurivillius, 1898

truncatus Dana, 1852

tuberculatus Bate, 1888 typus Milne Edwards

(H.), 1837

typus Milne Edwards

(H.), 1837

typus Milne Edwards

(H.), 1837

varians Leach, 1814

varians Leach, 1814

vittata Stimpson, 1860

zimmeri Balss, 1914
(2)

Palaemonetes trispinosus

Betaeus truncatus

Chorismus tuberculatus

Caridina typus

Oplophorus typus

Rhynchocinetes typus

Hippolyte varians

Palaemon varians

Hippolysmata vittata

Bathypalaemonella zimmeri
Genus of which species eited in Col. (1) is the type species

(3)

Desmocaris Sollaud, 1911

Betaeus Dana, 1852

Chorismus Bate, 1888

Caridina Milne Edwards

(H.), 1837

Oplophorus Milne

Edwards (H.), 1837

Rhynchocinetes Milne

Edwards (H.), 1837

Hippolyte Leach, 1814

Palaemonetes Heller, 1869

Hippolysmata Stimpson, 1860

Bathypalaemonella

Balss, 1914

6. In the case of fourteen of the genera enumerated in paragraph 3 of the present application, the name of the nominal species, which is the type species of the genus concerned is not accepted by specialists as the oldest available name for the taxonomic species represented by the nominal species in question. These cases are :-

Name of the genus
Name of nominal species which is the type species of the genus specified in the first column

(2)
Oldest available name for the species specified in the second column

(3)

Alope White, 1847 Alope palpalis White, 1847

Hippolyte spinifrons Milne Edwards (H.), 1837

Barbouria Rathbun, 1912 Barbouria poeyi Rathbun, Hippolyte cubensis Von 1912 Martens, 1872

Brachycarpus Bate, 1888 Brachycarpus savignyi Palaemon biunguiculatus Bate, 1888 Lucas, 1849 
Name of the genus

(1)
Name of nominal species which is the type species of the genus specified in the first column

(2)
Oldest available name for the species specified in the second column

Chlorocurtis

Chlorotocus Milne

Edwards (A.), 1882

1925

Chlorotocus gracilipes

Milne Edwards (A.), 1882

Chlorocurtis miser Kemp, Virbius (?) jactans

Nobili, 1904

Pandalus crassicornis

Costa, 1871

Latreutes Stimpson, 1860

Ligur Sarato, 1885

Hippolyte ensiferus Milne Palaemon fucorum Edwards (H.), $1837 \quad$ Fabricius, 1798

Ligur edwardsii Sarato, Palaemon ensiferus Risso, 1885 1816

Parapandalus Borradaile, Pandalus (Parapandalus) Plesionika spinipes Bate, 1899 serratifrons

Borradaile, 1899

Periclimenes Costa, 1844 Periclimenes insignis

Plesionika Bate, 1888

Costa, 1844

Plesionika uniproducta

Bate, 1888 1888

Alpheus amethysteus

Risso, 1826

Acanthephyra ensis Milne

Edwards (A.), 1881

Saron Thallwitz, 1891

Hippolyle gibberosus

Milne Edwards (H.),

Palaemon marmoratus

1837

Synalpheus Bate, 1888

Synalpheus falcatus Bate, Alpheus comatularum 1888

Haswell, 1882

Trachycaris Calman, 1906

Troglocaris Dormitzer,

1853

Platybema rugosus Bate, Hippolyte restricta Milne 1888

Troglocaris schmidti

Dormitzer, 1853

Edwards (A.), 1878

Palaemon anophthalmus

Kollar, 1848

7. The concrete proposals which I now submit for consideration are that the Commission should :-

(1) use its Plenary Powers :-

(a) to validate the emendation "Gnathophyllum" of the generic name originally published as Gnatophyllum by Latreille in

(b) to direct the family-group name HIPPOLYTIDAE Bate, 1888, be protected from its senior subjective synonyms LYSMATINAE 
Dana, 1852, and THORINAE Kingsley, 1878, in the manner specified in paragraph 2(11) of the present application ;

(c) to suppress the family-group name GoMPHonotidae Chace, 1936, for the purposes of the Law of Priority but not for those of the Law of Homonymy;

(2) rule that the specific name paronae Nobili, 1905, as published in the combination Latreutes paronae, being a name based upon the modern patronymic "Parona" is, under the Règles, subject to automatic correction to paronai ;

(3) place on the Official List of Generic Names in Zoology the one hundred and two generic names enumerated in paragraph 3 of the present application with the particulars there specified;

(4) place on the Official List of Specific Names in Zoology :-

(a) the eighty-eight specific names specified in paragraph 4 of the present application;

(b) the specific names of the fourteen nominal species listed in Column (3) in paragraph 5 of the present application ;

(5) place the under-mentioned generic names on the Official Index of Rejected and Invalid Generic Names in Zoology :-

(a) Atys Leach, 1815, Trans. linn. Soc. Lond. $11: 345$ (a junior homonym of Atys de Montfort, 1810 Conch. $2: 342$ );

(b) Balssiola Strand, 1922, Arch. Naturgesch. 88 (A4) : 142 (substitute name for Calmania Bouvier, 1909) (a junior objective synonym of Micratya Bouvier, 1913, Bull. Soc. ent. France $1913: 181$ );

(c) Calmania Bouvier, 1909, C. R. Acad. Sci. Paris 148 : 1730 (a junior homonym of Calmania Laurie, 1906, in Herdman, Rep. Ceylon Pearl Fish. 5 : 406);

(d) Doryphorus Norman, 1861, Ann. Mag. nat. Hist. (3)8: 27 (a junior homonym of Doryphorus Cuvier, 1829) ;

(e) Drimo Risso, 1826, Hist. nat. Europ. mérid. 5 : 70 (type species, by monotypy: Alpheus elegans Risso, 1816, Hist. nat. Crust. Nice:92) (a junior objective synomyn of Gnathophyllum Latreille, 1819);

(f) Gnatophyllum Latreille, 1819, Nouv. Dict. Hist. nat. (ed. 2) $30: 72$ (an Invalid Original Spelling of Gnathophyllum Latreille, 1819);

(g) Gomphonotus Chace, 1936, J. Wash. Acad. Sci. 26 : 25 (substitute name for Gonatonotus A. Milne Edwards (A.), 1881) (a junior objective synonym of Eugonatonotus Schmitt, 1926); 
(h) Gonatonotus Milne Edwards (A.), 1881, Ann. Sci. nat., Zool. (6)4(4) : 10 (a junior homonym of Gonatonotus Adams \& White, 1847) ;

(i) Hoplophorus Agassiz (J. L. R.), 1846, Nomencl. zool., Index Univ. : 185, 262 (an Invalid Emendation of Oplophorus Milne Edwards (H.), 1837) ;

(j) Nectocrangon Brandt, 1851, Middendorff's Reise Sibir. 2(1) : 114 (type species, by monotypy: Crangon lar Owen, 1839, Zool. Beechey's Voy. Blossom : 88) (a junior objective synonym of Argis Kröyer, 1842);

(k) Oedipus Dana, 1852, Proc. Acad. nat. Sci. Philad. 6 : 17 (a junior homonym of Oedipus Berthold, 1827, and replaced by Coralliocaris Stimpson, 1860);

(1) Ogyris Stimpson, 1860, Proc. Acad. nat. Sci. Philad. $1860: 36$ (a junior homonym of Ogyris Westwood, 1851, and replaced by Ogyrides Stebbing, 1914);

(m) Pasiphae Kröyer, 1845, Naturhist. Tidsskr. (n.s.) $1: 453$ (an Invalid Emendation of Pasiphaea Savigny, 1816);

(n) Pasiphaeia Faxon, 1895, Mem. Mus. comp. Zool. $18: 173$ (an Invalid Emendation of Pasiphaea Savigny, 1816) ;

(o) Regulus Dana, 1852, Proc. Acad. nat. Sci. Philad. $6: 18,27$ (a junior homonym of Regulus Cuvier (G.L.C.F.D.), 1800, and replaced by Thalassocaris Stimpson, 1860);

(p) Tridacnocaris Nobili, 1899, Ann. Mus. civ. Stor. nat. Genova $40: 235$ (substitute name for Anchistus Borradaile, 1898) (a junior objective synonym of Anchistus Borradaile, 1898);

(q) Xiphocaridina Bouvier, 1909, C. R. Acad. Sci., Paris 148 : 1729 (type species, by selection by Holthuis, 1955 (Zool. Verhand. Leiden $26: 21)$ : Ephyra compressa De Haan, 1844, Fauna japon., Crust. (6/7) : pl. 46, fig. 7) (a junior objective synonym of Paratya Miers, 1882).

(6) place the under-mentioned names on the Official List of Family-Group Names in Zoology :-

(a) Atyidae (correction by Dana (1852, Proc. Acad. nat. Sci. Philad. $6: 13,16)$ of ATYADEA) De Haan, 1849, Fauna japon., Crust. (6) : 168, 184 (type genus: Atya Leach, 1816);

(b) BResilmidae Calman, 1896, Trans. Roy. Irish Acad. $31: 7$ (type genus : Bresilia Calman, 1896);

(c) CaMPylonotidae Sollaud, 1913, Bull. Mus. Hist. nat., Paris 19 : 184 (type genus : Campylonotus Bate, 1888); 
(d) eugonatonotidae Chace, 1937, Proc. New Engl., zool. Cl. 16 : 15 (type genus : Eugonatonotus Schmitt) 1926, as proposed to be validated under the Plenary Powers;

(e) GLYPHocrangonidae Smith, 1884, Rep. U.S. Fish Comm. 10 : 364 (type genus: Glyphocrangon Milne Edwards (A.), 1881);

(f) Gnathophyllinae Dana, 1852, Proc. Acad. nat. Sci. Philad. 6 : 16 (type genus : Gnathophyllum Latreille, 1819) (first elevated to family rank as GNATHOPHYLLIDAE by Ortmann, 1890, Zool. Jahrb. Syst. 5 : 537)

(g) HIPpolytidae Bate, 1888, Rep. Voy. Challenger, Zool. 24 : xii, xli, 480, 503, 574, 576 (type genus : Hippolyte Leach, 1814) (a family-group name to be given preference under the Plenary Powers under (1)(b) above over the Family-Group names Lysmatinae Dana, 1852, Proc. Acad. nat. Sci. Philad. 6 : 16, 20 (type genus : Lysmata Risso, 1816) and thorinae Kingsley, 1878, Bull. Essex Inst. $10: 64$ (type genus: Thor Kingsley, 1878), by any author who may consider the genera Hippolyte Leach, Lysmata Risso, and/or Thor Kingsley as belonging to the same family-group taxon);

(h) nematocarcininae Smith, 1884, Rep. U.S. Fish Comm. $10: 368$ (type genus: Nematocarcinus Milne Edwards (A.), 1881) (first elevated to family rank as NEMATOCARCINIDAE by Smith, 1886, Rep. U.S. Fish Comm. 13 : 608, 619, 664)

(i) oplophorinae Dana, 1852, Proc. Acad. nat. Sci. Philad. 6 : 18, 27 (type genus: Oplophorus Milne Edwards (H.), 1837) (first elevated to family rank as OPLOPHORIDAE by Rathbun, 1902, Proc. U.S. nat. Mus. 24 : 904)

(j) Pasiphaeidae Dana, 1852, Proc. Acad. nat. Sci. Philad. 6 : 13, 18 (type genus: Pasiphaea Savigny, 1816);

(k) PSALIDOPODIDAE Wood Mason \& Alcock, 1892, Ann. Mag. nat. Hist. (6)9:265 (type genus: Psalidopus Wood Mason \& Alcock, 1892) ;

(l) RHYNChocinetidae Ortmann, 1890, Zool. Jahrb. Syst. $5: 459$

(type genus : RHYNCHOCINETES Milne Edwards (H.), 1837);

(m) STylodactylidae Bate, 1888, Rep. Voy. Challenger, Zool. 24 : 481, 850 (type genus: Stylodactylus Milne Edwards (A.), 1881);

(n) THALASSOCARIDIDAE (correction by Holthuis, 1955 (Zool. Verhand. Leiden. $26: 12,128$ ) of thalassocaridae) Bate, 1888, Rep. Voy. Challenger, Zool. 24 : lxxvii, 481, 682 (type genus: Thalassocaris Stimpson, 1860);

(o) TYPHLOCARIDINae Annandale \& Kemp, 1913, J. asiat. Soc. Bengal (n.s.) 9(6) : 245 (type genus: Typhlocaris Calman, 1909); 
(7) place the under-mentioned names on the Official Index of Rejected and Invalid Family-Group Names in Zoology :-

(a) ATYADEA De Haan, 1849 (type genus: Atya Leach, 1816) (an Invalid Original Spelling for ATYIDAE)

(b) ATtidae Yu, 1936, Chin. J. Zool. $2: 88$ (an Erroneous Subsequent Spelling for atyidae De Haan, 1849);

(c) BRESILIDAE Kemp, 1910, Sci. Invest. Br. Ireland 1908(1):35 (an Erroneous Subsequent Spelling for BRESILIIDAE Calman,
1896);

(d) DRIMOIDAe Ortmann, 1896, Zool. Jahrb. Syst. $9: 425$ (type genus : Drimo Risso, 1826) (invalid because the type genus of the family so named has, as its type species, Alpheus elegans Risso, 1816, which is also the type species of Gnathophyllum Latreille, 1819 (a name placed on the Official List of Generic Names in Zoology under (2) above), which is the type of the family GNATHOPHYLLIDAE Dana, 1852 (a name placed on the Official List of Family-Group Names in Zoology under (6)(f) above);

(e) gomphonotidae Chace, 1936, J. Wash. Acad. Sci. $26: 25$ (type genus; Gomphonotus Chace, 1936) (suppressed under the Plenary Powers under (1)(c) above);

(f) Gonatonotidae Gurney, 1941, J. linn. Soc. Lond., Zool. 41 : 122 (type genus: Gonatonotus Milne Edwards (A.), 1881) (invalid because the type genus of the family so named has, as its type species, Gonatonotus crassus Milne Edwards (A.), 1881, which is also the type species of Eugonatonotus Schmitt, 1926 (a name placed on the Official List of Generic Names in Zoology under (2) above), which is the type genus of the family EUGONATONOTIDAE Chace, 1937 (a name placed on the Official List of Family-Group Names in Zoology under (6)(d) above);

(g) HIPPolyidae Yu, 1935, Chin. J. Zool. $1: 43,45,47,49,51,53$ (an Erroneous Subsequent Spelling for HIPPOLYTIDAE Bate,
1888 );

(h) HYPPOLItIdae Dohrn, 1950, Pubbl. Sta. zool. Napoli 22 : 257, 259, 261, 263, 265, 267, 269, 271 (an Erroneous Subsequent Spelling for HIPPOLYTIDAE Bate, 1888);

(i) HOPLOPHORIDAe Faxon, 1895, Mem. Mus. comp. Zool. 18 : 159 (an Erroneous Subsequent Spelling for oplophorIDAE Dana,
1852);

(j) OPLOPHAIDAE Guiler, 1952, Rec. Queen Victoria Mus. Tasmania 3(3) : 35 (an Erroneous Subsequent Spelling for OPLOPHORIDAE Dana, 1852);

(k) PASIPHÄ̈IDAE Barnard, 1950, Ann. S. Afr. Mus. $38: 648$ (an Erroneous Subsequent Spelling for PASIPHAEIDAE Dana, 1852); 
(l) Pasiphaemdae Faxon, 1893, Bull. Mus. comp. Zool. $24: 208$ (an Erroneous Subsequent Spelling for PASIPHAEIDAE Dana, 1852);

(m) PASIPHAIDAE Smith, 1884, Rep. U.S. Fish Comm. $10: 381$ (an Erroneous Subsequent Spelling for PASIPHAEIDAE Dana, 1852);

(n) PASIPHAIDAE Wood Mason \& Alcock, 1893, Ann. Mag. nat. Hist (6) 11 : 161 (an Erroneous Subsequent Spelling of PASIPHAEIDAE Dana, 1852);

(o) PaSIPHeidae De Miranda y Rivera, 1933, Not. Res. Inst. Espan. Oceanogr. (2)67:6 (an Erroneous Subsequent Spelling of PASIPHAEIDAE Dana, 1852);

(p) RHincocynetidae Sharp, 1893, Proc. Acad. nat. Sci. Philad. 1893 : 118 (an Erroneous Subsequent Spelling of RHYNCHOCINETIDAE Ortmann, 1890);

(q) RHYNChocynetidae Borradaile, 1907, Ann. Mag. nat. Hist. (7) $19: 467$ (an Erroneous Subsequent Spelling of RHYNCHOCINETIDAE Ortmann, 1890);

(r) RYNCHOCINETIDAE Gurney, 1939, Ray Soc. $125: 72$ (an Erroneous Subsequent Spelling of RHYNCHOCINETIDAE Ortmann, 1890);

(s) thalassocaridae Bate, 1888, Rep. Voy. Challenger, Zool. 24 : lxxvii, 481, 682 (an Invalid Original Spelling of THALASSOCARIDIDAE Bate, 1888). 

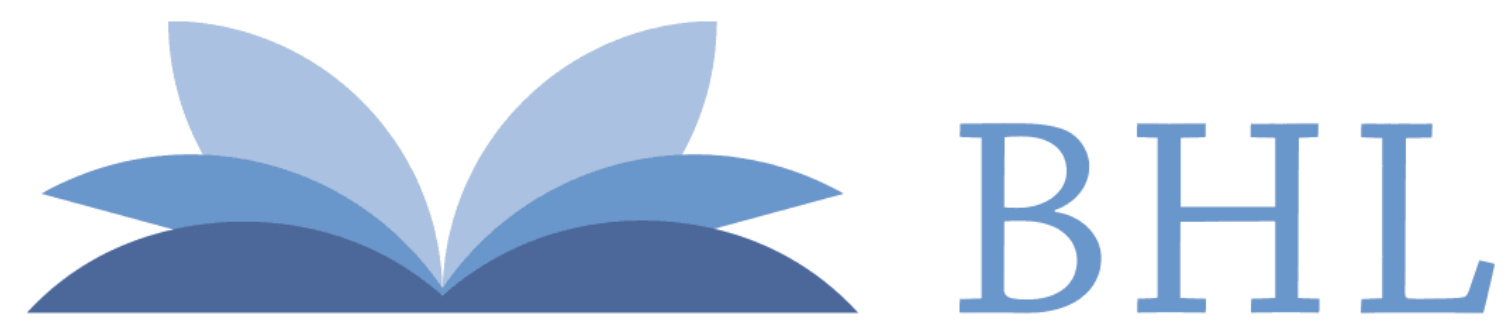

\section{Biodiversity Heritage Library}

Holthuis, L. B. 1955. "Proposed addition to the "Official List of Generic Names in Zoology" of the names of one hundred and two genera of Caridea (Class Crustacea, Order Decapoda), including proposals for the use of the plenary powers (a) to validate the emendation to "Gnathophyllum" of the generic name "Gnatophyllum" Latreille, 1814, and (b) to validate the family-group names "Hippolytidae" Bate, 1888 and "Eugonatonotidae" Chace, 1937." The Bulletin of zoological nomenclature 11, 204-228.

https://doi.org/10.5962/bhl.part.2844.

View This Item Online: https://www.biodiversitylibrary.org/item/44292

DOI: https://doi.org/10.5962/bhl.part.2844

Permalink: https://www.biodiversitylibrary.org/partpdf/2844

\section{Holding Institution}

Natural History Museum Library, London

\section{Sponsored by}

Natural History Museum Library, London

\section{Copyright \& Reuse}

Copyright Status: In copyright. Digitized with the permission of the rights holder.

License: http://creativecommons.org/licenses/by-nc-sa/3.0/

Rights: https://biodiversitylibrary.org/permissions

This document was created from content at the Biodiversity Heritage Library, the world's largest open access digital library for biodiversity literature and archives. Visit BHL at

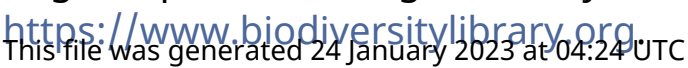

